\title{
DUREZA SUPERFICIAL DE RESINAS COMPOSTAS NANOPARTICULADAS E BULK FILL EXPOSTAS A SUPLEMENTOS NUTRICIONAIS
}

\author{
SURFACE HARDNESS OF COMPOSITE RESINS NANOPARTICULATE \\ AND BULK FILL EXPOSED TO NUTRITIONAL SUPPLEMENTS
}

\author{
Hamilton Bruno Santos COSTA'; Leonardo de Pádua Andrade ALMEIDA²; Miquéias Sousa SILVA; Alessandro Ribeiro \\ GONÇALVES ${ }^{4}$ \\ 1 - Graduação em Odontologia pela Universidade Federal do Piauí (UFPI) - 2018. \\ 2 - Graduação em Odontologia pela UFPI - 2017. \\ 3 - Técnico do laboratório de Engenharia de Materiais da UFPI. \\ 4 - Professor Adjunto do Departamento de Odontologia Restauradora da UFPI. Doutor em Reabilitação Oral - Universidade Estadual Paulista Júlio \\ de Mesquita Filho (UNESP).
}

\section{RESUMO}

Objetivo: Avaliar a microdureza superficial de duas resinas compostas após imersão controlada a dois suplementos nutricionais à base de carboidratos, desenvolvidos para indivíduos que praticam atividade esportiva. Materiais e Métodos: Utilizou-se as resinas compostas Filtek Z350 XT (3M ESPE); Filtek bulk Fill (3M ESPE) ambas na cor A3. Foram confeccionados 60 corpos-de-prova, 30 para cada material restaurador, os quais foram divididos nos seguintes grupos: Grupo 1 - controle; Grupo 2 imersão em $20 \mathrm{~mL}$ de Gatorade (AmBev) durante 5 minutos 3 vezes por dia; Grupo 3 - imersão em $30 \mathrm{~g}$ de gel de carboidrato Carbo Gel (Vox Suplementos) durante 5 minutos 3 vezes por dia. Em todos os grupos o período de reposição foi de 15 dias. Durante os intervalos, as resinas ficaram armazenadas em saliva artificial a $37^{\circ} \mathrm{C}$. Foi realizado o teste de microdureza Vickers com base na norma ISO 4049 com o auxílio de um Microdurometro Vickers - Buehler - Lake Bluff, Illinois/USA (MMT-3) com a carga de 50 gf por 30 segundos, foram realizadas 5 endentações por corpo-de-prova, uma no centro e quatro nas periferias. A média dos valores de dureza foi comparada usando o teste ANOVA e o pós-teste de Tukey, em nível de significância de $5,0 \%$. Resultados: Todos os corpos de prova que ficaram imersos em Gatorade e Gel de carboidrato apresentaram valores de microdureza estatisticamente menores, quando comparados aos grupos controle. Conclusão: A imersão de resinas compostas em suplementos alimentares Gatorade (Ambev) e Carbo Gel (Vox Suplementos) diminui significativamente sua dureza superficial.

Palavras-chave: Resinas compostas; Dureza; Suplementos Nutricionais; Materiais dentários.

\section{INTRODUÇÃO}

O consumo de bebidas energéticas na prática de atividades esportivas tem se tornado cada vez mais frequente. Embora o propósito dessas bebidas seja melhorar o desempenho, a resistência física e evitar desidratação dos indivíduos envolvidos em atividades físicas, eles estão sendo amplamente consumidos pela população em geral. No entanto, estudos anteriores mostram que essas bebidas causam erosão dentária, devido à sua acidez, podendo também ser prejudicial para as propriedades dos materiais restauradores ${ }^{1}$.

As restaurações diretas em resina composta são frequentes na odontologia moderna. Elas apresentam a vantagem de serem estéticas, possuírem adesão à estrutura dentária e terem um custo reduzido quando comparadas com as restaurações indiretas ${ }^{2}$.

Para ter sucesso clínico, as resinas compostas dentárias devem ser duráveis e ter alto grau de resistência ao desgaste na cavidade oral ${ }^{3,4}$. Elas são expostas contínua ou intermitentemente a agentes químicos encontrados na saliva, comidas e bebidas. Tal exposição pode amolecer a matriz da resina composta resultando em perda de partículas. Assim, as propriedades mecânicas das resinas compostas são influenciadas não somente por sua composição química, mas também pelo meio ambiente a que estão expostas ${ }^{5,6}$.

Honório et al. ${ }^{7}$ (2008) relacionaram o consumo de comidas e bebidas ácidas com desgaste erosivo de materiais restauradores. Levando-se em consideração que vários dos suplementos ou estimulantes utilizados na prática de exercícios físicos são substâncias ácidas, eles podem modificar as superfícies dos materiais restauradores causando algum nível de degradação.

Deve ser analisado se o consumo frequente de suplementos alimentares pode causar algum dano aos materiais restauradores diretos. Uma das formas de avaliar esse dano é através da microdureza superficial, que é um importante indicador da resistência mecânica dos materiais restauradores ${ }^{1}$. Assim, o objetivo desse trabalho foi mensurar a microdureza superficial de duas resinas compostas após serem expostas a dois suplementos nutricionais à base de carboidratos desenvolvidos para atividade esportiva. 


\section{MATERIAIS E MÉTODOS}

Para a realização do trabalho foram utilizadas as resinas compostas Filtek Z350 XT (3M ESPE); Filtek Bulk Fill (3M ESPE), ambas na cor A3.

Os corpos de prova foram confeccionados utilizando uma matriz de teflon com uma perfuração central circular de $5 \mathrm{~mm}$ de diâmetro e $2 \mathrm{~mm}$ de espessura (Figura 1).

As resinas foram inseridas na matriz com o auxílio de uma espátula para inserção de resina dupla $\mathrm{n}^{\circ} 1$ (Golgran) em incremento único. Sobre a superfície superior foi posicionada uma lâmina de vidro transparente pressionando manualmente o incremento, utilizou-se o aparelho foto polimerizador Kondortech modelo CLK200 (Kondortech, São Carlos, Brasil) durante 40 segundos. Antes do seu uso o aparelho foi testado em um radiômetro (Ecel RD-7 Digital) para ter certeza de sua eficácia, encontrando-se uma intensidade de $572 \mathrm{~mW} / \mathrm{cm} 2$. Após a foto ativação, as amostras foram imersas em saliva artificial por 24 horas em uma estufa a $37^{\circ} \mathrm{C}$.

Foram confeccionados 60 corpos-de-prova, 30 para cada material restaurador, os quais foram divididos nos seguintes grupos: Grupo 1 (controle) - imersão em saliva artificial por 15 dias. Grupo 2 - imersão em $20 \mathrm{ml}$ de Gatorade (AmBev) durante 5 minutos 3 vezes por dia, após cada imersão, a amostra era lavada com água destilada e estocada em saliva artificial em estufa a $37^{\circ} \mathrm{C}$; o processo foi repetido por 15 dias; Grupo 3 - imersão em $30 \mathrm{~g}$ de gel de carboidrato Carbo Gel (Vox Suplementos) durante 5 minutos 3 vezes por dia. Após cada imersão, a amostra era lavada com água destilada e estocada em saliva artificial em estufa a $37^{\circ} \mathrm{C}$; o processo foi repetido por 15 dias. Para todos os grupos, a saliva artificial foi trocada a cada cinco dias. $\mathrm{O} \mathrm{pH}$ de todas as substâncias foi analisado com o Medidor de $\mathrm{pH}$ e temperatura EC, TDS (Hanna). Em seguida, foi realizado o teste de microdureza Vickers com base na norma ISO 4049 com o auxílio de um Microdurometro Vickers - Buehler - Lake Bluff, Illinois/ USA (MMT-3) sob uma carga de $50 \mathrm{gF}$ por 30 segundos. Realizando 5 endentações de microdureza por corpo-de-prova, uma no centro e quatro nas periferias. Os dados obtidos a partir das médias foram submetidos à análise de variância estatística ANOVA, seguida pelo teste Tukey em nível de 5,0\% de significância.

\section{RESULTADOS}

Os dados foram submetidos ao teste de normalidade e verificou-se que a distribuição da amostra foi normal. A análise estatística foi realizada no software SPSS, 2017 para Windows. Constatada distribuição normal foi utilizado o teste paramétrico ANOVA $(p \leq 0,05)$, demostrou que os fatores analisados, solução e resina, isoladamente, assim como a interação entre os fatores apresentaram efeito sobre a microdureza. Para verificação das diferenças utilizou-se o teste de Tukey.

Os resultados obtidos mostraram que as resinas Z350 XT (3M ESPE) e Bulk Fill (3M ESPE) apresentaram diminuição significativa da microdureza quando imersas em Gatorade e Gel de carboidrato, sendo que, quando imersa em Gatorade, a Resina Bulk Fill (3M ESPE) apresentou um valor de dureza estatisticamente inferior quando comparada à resina Z350 XT (3M ESPE). No presente estudo, os valores de $\mathrm{pH}$ obtidos foram de 3,17 (Gatorade) e 4,0 (Gel de carboidrato), coerentes com estudos anteriores em que resultados semelhantes foram encontrados, os quais utilizaram bebidas energéticas esportivas que continham baixo $\mathrm{pH}^{23}$.

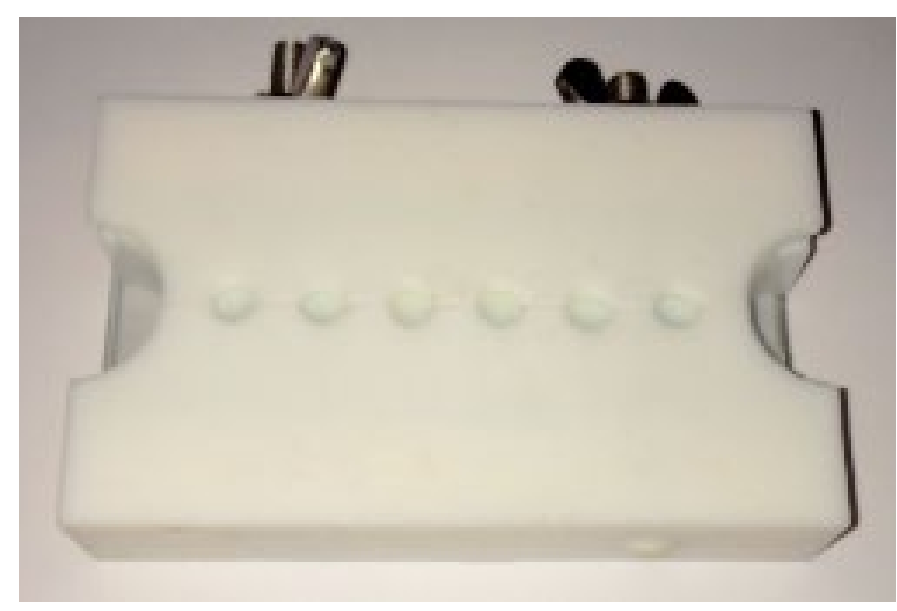

Figura 1 - Matriz de Teflon

Tabela 1 - Comparação entre as médias de microdureza dos grupos após imersão com seus respectivos desvios padrões.

\begin{tabular}{llll}
\hline & G1- Controle & G2- Gatorade & G3- Gel de carboidrato \\
\hline Z350 & $70,36 \pm 1,37 \mathrm{Aa}^{*}$ & $64,32 \pm 2,07 \mathrm{Ab}^{*}$ & $60,22 \pm 1,46 \mathrm{Ac}^{*}$ \\
\hline Bulk Fill & $68,95 \pm 1,71 \mathrm{Aa}^{*}$ & $61,03 \pm 1,67 \mathrm{Bb}^{*}$ & $58,74 \pm 1,77 \mathrm{Ac}^{*}$ \\
\hline
\end{tabular}

*Letras minúsculas diferentes na mesma linha e letras maiúsculas diferentes na mesma coluna significam diferença estatística significativa pelo Pós- teste de Tukey após ANOVA $(P<0,05)$.

\section{DISCUSSÃO}

As bebidas isotônicas são habitualmente consumidas por adolescentes e adultos jovens. Possuem concentração de substâncias ou minerais semelhantes às encontradas nos fluidos orgânicos e o balanço entre os eletrólitos (minerais) evita a desidratação durante a prática de esportes. Tais bebidas são tipicamente carbonatadas, de elevada acidez, com baixo conteúdo de carboidratos, variando de 6 a $8 \% 8$.

O consumo de bebidas energéticas durante a prática esportiva é, muitas vezes, maior do que o de água, devido à sua palatabilidade, caracterizada pela temperatura da bebida, "doçura", sabor e intensidade do gosto9.

A erosão dental começa com a desmineralização das camadas superficiais do esmalte, podendo evoluir para uma perda considerável de estrutura dentária. Qualquer substância ácida com $\mathrm{pH}$ inferior ao crítico para a dissolução do esmalte $(\mathrm{pH}<5,5)$ e dentina $(\mathrm{pH}<6,5)$ pode dissolver os cristais de hidroxiapatita ${ }^{10}$. É importante acrescentar que quando a saliva está subsaturada de hidroxiapatita, ainda pode permanecer supersaturada de fluorapatita (quando há presença de flúor na boca). No $\mathrm{pH}=4$, a saliva está subsaturada de ambas as apatitas e, portanto, perde a capacidade mineralizante ${ }^{11}$.

Entre todos os materiais restauradores disponíveis, as resinas compostas se tornaram a primeira escolha de material restaurador, devido a melhorias em suas propriedades físicas, mecânicas e ópticas e facilidade no manuseio clínico. Diferentes cargas e sistemas de monômeros são modificados ou adicionados para obter sucesso clínico das restaurações.

Com o aumento do uso de materiais restauradores estéticos, surgiram também preocupações acerca da degradação destes materiais, justificando a realização de diversos experimentos sobre o assunto ${ }^{12}$. Com isso, as propriedades físicas, mecânicas 
e ópticas das resinas compostas expostas a bebidas energéticas esportivas começaram a ser estudadas.

Abu-Bakr et al. ${ }^{13}(2000)$ pesquisaram o efeito de bebidas com baixo $\mathrm{pH}$ na dureza superficial de materiais restauradores, relatando que um compômero imerso em substâncias ácidas, como o refrigerante, exibiu dureza significativamente menor que um compômero imerso em água destilada pelo mesmo período.

Nicholson et al. ${ }^{14}$ (2003) analisaram bebidas esportivas energéticas e observaram que os níveis de $\mathrm{pH}$ tiveram uma variação entre 2,67 e 3,79. Com isso eles concluíram que a imersão de materiais restauradores durante uma semana, resultou em diminuição da dureza superficial, sugerindo uma susceptibilidade das resinas a corroerem sob condições ácidas.

No estudo de Cavalcanti et al. ${ }^{15}$ (2010), foram analisadas bebidas energéticas (Gatorade e Powerade) utilizadas em atividades esportivas, e confirmou-se que elas apresentam $\mathrm{pH}$ baixo, variando de 2,98 a 3,07.

Segundo Moroz et al. ${ }^{16}(2010)$ e Cavalcanti et al. ${ }^{15}$ (2010) essas soluções têm $\mathrm{pH}$ potencialmente erosivo para o esmalte dental e são capazes de causar alterações na microdureza de resinas compostas.

Uma grande variedade de substâncias está presente no ambiente oral, água, saliva, ácidos, bases, sais e álcoois e têm sido relacionadas à redução da dureza e resistência à flexão. Diferenças na suscetibilidade ao amolecimento e degradação podem ser atribuídas à interação entre as bebidas ácidas e os componentes químicos das resinas compostas ${ }^{17}$.

De acordo com Moraes et al. ${ }^{18}$ (2010), a dureza superficial dos materiais restauradores diminui entre a primeira semana e o primeiro mês de imersão em soluções de baixo $\mathrm{pH}$. A deterioração de materiais resinosos provavelmente ocorre por causa da absorção de água, onde a presença desta pode amolecer a resina por inchaço, afetando a rede polimérica e reduzindo as forças de atrito entre as cadeias. Além disso, resinas têm alta solubilidade em soluções de baixo $\mathrm{pH}$, levando à erosão e dissolução da superfície, suavização da matriz e perda de íons estruturais ${ }^{14,19,20}$.

$\mathrm{O}$ valor baixo do $\mathrm{pH}$ pode afetar a resistência de materiais restauradores, pela decomposição da matriz orgânica e da interação com as partículas de carga, podendo assim, resultar em alterações na microdureza do material restaurador ${ }^{21}$.

De acordo com os resultados obtidos, todos os corpos de prova que ficaram imersos em Gatorade e Gel de carboidrato apresentaram valores de microdureza estatisticamente menores, quando comparados ao grupo controle. Este achado concorda com o estudo de Santos et al. ${ }^{21}$ (2014); eles analisaram a influência de bebidas energéticas com um baixo $\mathrm{pH}$ sobre a microdureza de resinas compostas. Verificaram que, após um ciclo de imersão, houve uma diminuição estatisticamente significativa da microdureza.

O presente estudo analisou in vitro a microdureza Vickers de dois materiais restauradores estéticos após a exposição a suplementos à base de carboidratos. A dureza dos materiais é uma das mais importantes propriedades e correlaciona-se bem com compressão, resistência ao amolecimento intraoral e grau de conversão. Um baixo valor de dureza superficial é amplamente relacionado à inadequada resistência ao desgaste, o que pode levar à fadiga e ao fracasso da restauração. Consequentemente, a curto ou a longo prazo, estas condições podem ter um efeito deletério diferente sobre a rede polimérica, modificando sua estrutura fisicamente e quimicamente.

A Filtek bulk Fill (3M ESPE) é uma resina nanoparticulada que pode ser inserida e polimerizada em até $5 \mathrm{~mm}$ de profundidade, devido a um sistema de monômeros resinosos que apresentam alívio de tensões de contração de polimerização e propriedades físicas otimizadas, AFM (monômero para alívio dinâmico de tensões de contração de polimerização), AUDMA, UDMA e 1,12-dodecano-DMA. No presente estudo a resina bulk fill apresentou uma diminuição estatisticamente significante da microdureza em relação à Z350 quando imersa em Gatorade. Segundo Yap et al..$^{22}$ (2001) este fator pode estar relacionado com a presença de GMA e UDMA, que são mais suscetíveis ao amolecimento após exposição a agentes químicos.

A Filtek Z350 XT (3M ESPE) também é uma resina nanoparticulada com algumas diferenças na matriz orgânica, contém BIS-GMA, BIS-EMA e UDMA, com pequenas quantidades de TEGDMA. O alto número de ligações duplas resultantes por unidade de peso, numa estrutura flexível, tornou possível obter uma alta conversão de ligações duplas durante a polimerização. $\mathrm{O}$ baixo peso molecular e o elevado número de ligações duplas resultantes por unidade de peso criam um alto grau de encadeamento cruzado, produzindo assim uma resina muito rígida. Devido a Z350 XT (3M ESPE) conter Bis-EMA e TEGDMA, provavelmente proporcionou melhor resistência contra o ataque químico ${ }^{18}$.

Baseado nos resultados desse estudo e nos relatos da literatu$\mathrm{ra}^{14,16,17,19,20}$ de que materiais resinosos imersos em bebidas ácidas têm uma alta solubilidade, e esta causa erosão e dissolução da superfície, afetando a dureza e integridade superficial; recomendamos que o uso de bebidas energéticas esportivas à base de carboidratos deve ser moderado, pois, ao serem enjeridos em excesso podem reduzir a dureza superficial das resinas estudas, o que pode afetar a longevidade das restaurações.

\section{CONCLUSÃO}

A imersão aos suplementos alimentares Gatorade (Ambev) e Carbo Gel (Vox Suplementos) reduziram a dureza superficial das resinas compostas estudadas.

\section{REFERÊNCIAS}

01. Erdemir U; Yldiz E; Eren MM; Ozel S. Surface hardness of different restorative materials after long-term immersion in sports and energy drinks. Dent Mater J. 2012; 31(5): 729-736.

02. Benetti AR, Jesus VCR, Martinelli NL, Pascotto RC, Poli-Frederico RC. Colour stability, staining and roughness of silorane after prolonged chemical challenges. J Dent. 2013; 41(12): 1229-35.

03. Okada K, Tosaki S, Hirota K, Hume WR. Surface hardness change of restorative filling materials stored in saliva. Dent Mater. 2001; 17(1): 34-39.

04. Suese K, Kawazoe T. Wear resistance of hybrid composite resin for crown material by the two-body sliding test. Dent Mater J. 2002; 21(3): 225-237.

05. Asmussen E. Softening of BISGMA-based polymers by ethanol and by organic acids of plaque. Scand J Dent Res.1984; 92(3): 247-261.

06. Wu W, Mckinney JE. Influence of chemicals on wear of dental composites. J Dent Res. 1982; 61(10): 1180-1183.

07. Honório HM, Rios D, Fracisconi LF, Magalhâes AC, Machado MA, Buzlafa, ALAF MA. Effect of prolonged erosive $\mathrm{pH}$ cycling on different restorative materials. J Oral Rehabil. 2008; 35(12): 947-53. 
08. Petrus RR, Faria JAF. Processamento e avaliação de estabilidade de bebida isotônica em garrafa plástica. Ciênc. Tecnol. Aliment. 2005; 25(3): 518-24.

09. Guerra I. Importância da alimentação e da hidratação do atleta. R. Min. Educ. Fís. 2004; 12(2): 159-73.

10. Lima HMR, Lima LR, Galvão FFDSP. Consumo infantil de bebidas lácteas: sólidos solúveis totais (Brix) e pH. Odontol. Clin. Cient. 2011; 10(3): 237-41.

11. Larsen MJ, Bruun C. Esmalte-saliva: reações químicas inorgânicas. In: Thylstrup A, Fejerkov O. Tratado de cariologia. 2nd ed. RJ, 1998. p. 169-93.

12. Bagheri Y, Gokc K, Kazak M. Subsurface degradation of resin based composites. Dental Materials. 2007; 23(8): 944-951.

13. Abu-Bakr N. et al. Color stability of compomer after immersion in various media. J Esthet Dent. 2000; 12(5): 258-63.

14. Nicholson JW, Gjorgievska E, Bajraktarova B, McKenzie MA. Changes in properties of polyacid-modified composite resins (compomers) following storage in acidic solutions. J Oral Rehabil. 2003; 30(6): 601-607.

15. Cavalcanti AL, Xavier AFC, Souto RQ, Oliveira MC, Santos JA, Vieira FF. Avaliação in vitro do potencial erosivo de bebidas isotônicas. Rev Bras Med Esporte. 2010; 16(6): 455-8.

16. Moroz LT, Alves BL, Luciano MP, Gibson LK, Pereira S. Influência de substâncias com pH ácido sobre a microdureza de resinas compostas. Stomatos. 2010; 16(30): 21-32.
17. Finer $\mathrm{Y}$, Santerre JP. The influence of resin chemistry on a dental composite's biodegradation. J Biomed Mater Res A. 2004; 69(2): 233-246.

18. Moraes RR, Marimon JL, Schneider LF, Sinhoreti MA, CorrerSobrinho L, Bueno M. Effects of 6 months of aging in water on hardness and surface roughness of two microhybrid dental composites. J Prosthodont. 2008; 17(4): 323-326.

19. Catelan A, Briso AL, Sundfeld RH, Dos Santos PH. Effect of artificial aging on the roughness and microhardness of sealed composites. J Esthet Restor Dent. 2010; 22(5): 324-330.

20. Okte Z, Villalta P, García-Godoy F, Lu H, Powers JM. Surface hardness of resin composites after staining and bleaching. Oper Dent. 2006; 31(5): 623-628.

21. Santos JL, Cassaro SE, Santos JR, Scalco VF, Barbosa KC, Lauris JRP, et.al. Influência de diferentes soluções químicas na microdureza de resinas compostas. UNOPAR Científica Ciências Biológicas e da Saúde. 2014; 7(1): 49-54.

22. Yap AU, Tan SH, Wee SS, Lee CW, Lim EL, Zeng KY. Chemical degradation of composite restoratives. J Oral Rehabil. 2001; 28(11): 1015-1021.

23. Meenakumari C, Bhat KM, Bansal R, Singh N. Evaluation of Mechanical Properties of Newer Nanoposterior Restorative Resin Composites: An In vitro Study. Contemp Clin Dent. 2018; 9(Suppl 1): S142-S146.

\section{ABSTRACT}

Objective: To evaluate the surface microhardness of two composite resins when exposed to two nutritional supplements based on carbohydrates developed for sports activity. Materials and Methods: Filtek Z350 XT composite resins (3M ESPE) were used; Filtek bulk Fill (3M ESPE) both in color A3. Sixty specimens were prepared, 30 of which were for each restorative material, divided into the following groups: Group 1 (control); Group 2 immersion in $20 \mathrm{~mL}$ of Gatorade (AmBev) for 5 minutes 3 times per day; Group 3 - immersion in $30 \mathrm{~g}$ Carbo Gel Carbohydrate Gel (Vox Supplements) for 5 minutes 3 times a day, the process was repeated for 15 days. The Vickers micro hardness test was performed based on ISO 4049 with the aid of a Vickers - Buehler
- Lake Bluff, Illinois / USA (MMT - 3) Microduometer with a load of $50 \mathrm{gF}$ for 30 seconds. Performing 5 indentations per body of evidence, one in the center and four in the peripheries. Data were submitted to analysis of statistical variance ANOVA, followed by Tukey test at a level of $5.0 \%$ of significance. Results: All specimens that were immersed in Gatorade and Carbohydrate Gel had statistically lower microhardness values when compared to the control group. Conclusions: The use of Gatorade (Ambev) and Carbo Gel supplements (Vox Supplements) decreased the surface hardness of the studied resins.

Keywords: Composite Resins; Hardness; Dietary Supplements; Dental Materials.

\section{AUTOR PARA CORRESPONDÊNCIA}

Hamilton Bruno Santos Costa

Campus Universitário Ministro Petrônio Portella-

Departamento de Odontologia Restauradora, Bairro Ininga/

CEP: 64049-550. Teresina-PI - Brasil.

Telefone: (86) 99813-8891

E-mail: hamiltoncosta1@live.com 